ACTA BOTANICA MALACITANA es una revista de carácter internacional y de periodicidad anual que tiene por objeto la transmisión de los resultados de trabajos originales relacionados con cualquier campo de la fitología y, especialmente, aquellos dedicados a la sistemática, corología y ecología, aplicados tanto a las disciplinas de la criptogamia como de la fanerogamia.

Accredited with the International Association for Plant Taxonomy for the purpose of registration of all new plant names.

ABM es editada por el Área de Botánica del Departamento de Biología Vegetal y publicada por el Servicio de Publicaciones e Intercambio Científico de la Universidad de Málaga.

Editor especial para este volúmen: Ángel Enrique SALVO TIERRA (Universidad de Málaga)

\title{
Comité Asesor
}

Alba Luz ARBELÁEZ (Universidad de Antioquía, Colombia), David BARRINGTON (Universidad de Massachusetts en Amherst, USA); Noelia HIDALGO TRIANA (Universidad de Málaga, España); Jose María GABRIEL Y GALÁN (Universidad Complutense de Madrid, España); Ruth Karina HERNÁNDEZ (Universidad Nacional Autónoma de Honduras, UNAH); Regina HIRAI (Instituto de Botânica, Centro de Investigación de Plantas Vasculares Brasil São Paulo); Emilio LAGUNA LUMBRERAS (Centro para la Investigación y Experimentación Forestal - Servicio de Vida Silvestre. Valencia, España); Blanca LEÓN (Universidad Nacional Mayor de San Marcos, Perú); Fernando MATOS (Universidade Federal do Paraná, Brasil); Aniceto MENDOZA RUIZ (Universidad Autónoma Metropolitana, México); Julián MOSTACERO GIANNANGELI (Universidad Central de Venezuela); Mónica PALACIOS-RÍOS (Instituto de Ecología, A.C. - Inbioteca, Universidad Veracruzana, México); Carmen PRADA MORAL (Universidad Complutense de Madrid, España); Alexander ROJAS ALVARADO (Universidad Nacional de Costa Rica); Germinal ROUHAN (Institut de Systématique, Evolution, Biodiversité, Muséum National d'Histoire Naturelle, France); Daniel TEJERO DÍEZ (Universidad Nacional Autónoma de México); Weston TESTO (Universidad de Vermont, USA); Luz Amparo TRIANA MORENO (Universidad de Caldas, Colombia)

\section{Secretario de redacción y responsable de versión electrónica: José GARCÍA SÁNCHEZ y E. BAÑARES}

\section{Comité Editorial}

Elena BAÑARES ESPAÑA (Editora en Jefe); Baltasar CABEZUDO ARTERO; Antonio FLORES MOYA; M. Mar TRIGO; Andrés V. PÉREZ LATORRE; Teresa NAVARRO DEL ÁGUILA; Marta RECIO; Antonio GALÁN DE MERA

\section{Maquetación: Ma Dolores TIRADO \\ Administración: Eva LINERO}

Fotografía portada:

Pteris arbelaeziana A. Rojas

Nueva especie descrita en este volumen

COSTA RICA. San José: Pérez Zeledón, San Gerardo de Rivas, Parque Nacional Chirripó.

Alexánder F. Rojas

Filigranas portada:

- Cystoseira tamariscifolia (Huds.) Papenf. Tomado de Flora Phycologica Iberica (Vol. 1)

- Hedwigia ciliata var. leucophaea Bruch \& Schimp. Tomado de Flora Briofítica Ibérica (Vol. V)

- Notholaena marantae (L.) Desv. subsp. marantae. Tomado de Flora Iberica (Vol. 1)

- Abies pinsapo Boiss. Tomado de Flora Iberica (Vol. 1)

- Limonium malacitanum Díez Garretas. Tomado de Trabajos y Monografías 2 (UMA)

- Posidonia oceanica (L.) Delile. Tomado de Flora Iberica (Vol. 17)

ÁREA DE BOTÁNICA. DEPARTAMENTO DE BIOLOGÍA VEGETAL

Publicada en diciembre de 2017 FACULTAD DE CIENCIAS

AVDA. LOUIS PASTEUR, 31

UNIVERSIDAD DE MÁLAGA

Tel. 952133342

e-mail: abm@uma.es

SERVICIO DE PUBLICACIONES

E INTERCAMBIO CIENTÍFICO

UNIVERSIDAD DE MÁLAGA

29071, MÁLAGA (ESPAÑA)

I.S.S.N. 0210 - 9506. Depósito Legal: MA. 338 - 1979.

I.S.S.N. 2340-5074 (Versión electrónica)

Impresa en Andalucía 